East Asian Mathematical Journal Vol. 30 (2014), No. 1, pp. 051-062

http://dx.doi.org/10.7858/eamj.2014.005

\title{
DYNAMICAL COCYCLES ASSOCIATED WITH CERTAIN NON-MEDIAL LEFT-DISTRIBUTIVE QUASIGROUPS
}

\author{
Hyun-Jong Song* And Hyo-SeOB Sim
}

\begin{abstract}
The purpose of this paper is to explicitly present AndruskiewitschGraña's dynamical cocycles associated with the two known non-medial left-distributive quasigroups of order 15 which are extensions of the dihedral quandle of order 3 by those cocycles.
\end{abstract}

\section{Introduction}

In knot theory, quandles were considered by G. Wraith and J. Conway in 1959 as a generalization of a group with the binary operation given by conjugation, and further developed by D. Joice [7] in 1980 for invariants of knots. In particular, connected finite quandles receive attentions for generalization of the classical Fox's $n$-colorings of knots [12].

A family of connected finite quandles were already investigated in the other area of mathematics with terms such as distributive (both left and right) or leftdistributive quasigroups which include all connected finite Alexander quandles, a major class of finite quandles in knot theory.

Since it is known that for order $<81$ any distributive quasigroup is necessarily medial (c.f. [10] or [8]), one may ask if there are non-medial left-distributive quasigroups of order $<81$. We take this opportunity to keep tracking of the status of the above question. Around 2008, Baik-Sim-Song circulated an unpublished manuscript [2] reporting existence of two non-medial left-distributive quasigroups of order 15. One of them was referred to as Galkin and denoted by $G_{15}$; the other Stanovsky and $S_{15}$. Indeed Galkin [5] showed $G_{15}$ in his survey article of quasigroups. On the other hand Stanovsky [11] constructed $S_{15}$ by using a computer. Later on Clark and et al.[3] extended Galikin's construction; in particular they pointed out that there are only two Galkin type quasigroups $G\left[Z_{5},[0]\right)$ and $G\left[Z_{5},[1]\right)$ of order 15 . Finally Vendramin [14] and Vlachy [15]

Received August 9, 2013; Accepted December 30, 2013.

2000 Mathematics Subject Classification. 57M27; 17B37, 20 N05.

Key words and phrases. finite connected quandles, dynamical cocycles, left-distributive quasigroups.

* This work was supported by a Research Grant of Pukyong National University (2013 year). 
independently proved that there are no more non-medial left-distributive quasigroups of order 15 except for $G\left[Z_{5},[0]\right)$ and $G\left[Z_{5},[1]\right)$. In his survey article [4] Elhamdadi reviewed on the progress mentioned in the above. The purpose of this paper is to explicitly present Andruskiewitsch-Graña's dynamical cocycles [1] associated with $G_{15}=G\left(Z_{5},[0]\right)$ and $S_{15}=G\left(Z_{5},[1]\right)$, which are extensions of the dihedral quandle of order 3 (referred to as the Tait quandle for short) by those cocycles.

\section{Preliminaries}

In this paper we adopt the left-handed definition of a quandle in order to be in concordance with treatment of previous results used in the work on which our research is based.

We recall some terminologies for binary systems.

A groupoid $(X, *)$, a non-empty set $X$ with a binary operation $*$, is said to be:

(1) idempotent if for each $x \in X x * x=x$,

(2) left-invertible if for each $x \in X$ the function $L_{x}: X \rightarrow X$ defined by $L_{x}(y)=x * y(y \in X)$ is bijective,

(3) right-invertible if for each $y \in X$ the function $R_{y}: X \rightarrow X$ defined by $R_{y}(x)=x * y(x \in X)$ is bijective,

(4) left-distributive if for each $x, y, z \in X x *(y * z)=(x * y) *(x * z)$,

(5) right-distributive if for each $x, y, z \in X(x * y) * z=(x * z) *(y * z)$,

(6) distributive if both left- and right-distributive,

(7) medial if for each $x, y, z, w \in X(x * y) *(z * w)=(x * z) *(y * w)$.

An idempotent, left-invertible and left-distributive groupoid is called a quandle, and a left- as well as right- invertible groupoid is called a quasigroup.

For a quandle $(Q, *)$, we call each bijection $L_{x},(x \in Q)$ a left-translation which is an automorphisms of $Q$ due to left-distributivity. The group generated by $\left\{L_{x} \mid x \in Q\right\}$ is called the inner automorphism group of $Q$ and denoted by $\operatorname{Inn}_{*}(Q)$. A quandle $(Q, *)$ is said to be connected if $\operatorname{Inn}_{*}(Q)$ acts transitively on $Q$, i.e., for each $x, y \in Q$ there exists $\phi \in \operatorname{Inn}_{*}(Q)$ such that $\phi(x)=y$.

In the sequel we assume that all groupoids we deal with are finite. From Lemma 2.1 to Theorem 2.4 we recall some well-known facts in quasigroup theory.

Lemma 2.1. If $(X, *)$ is a left-distributive quasigroup then, $(X, *)$ is idempotent.

Remark. A left-distributive quasigroup is referred to as a Latin quandle in some literatures due to the fact that a multiplication table of a quasigroup of order $n$ constitutes a Latin square, namely an $n \times n$ array (matrix in which each of the $n^{2}$ cells contain a number from $I=\{1,2, \cdots, n\}$ so that each number of $I$ occurs just once in each row and once in each column. 
Lemma 2.2. If $(X, *)$ is a medial idempotent quasigroup, then $(X, *)$ is a distributive quasigroup.

However the converse is not true in general as stated in the introduction.

Lemma 2.3. Let $A=(A,+)$ be an abelian group with two commuting automorphisms $f, g$ of $A$ and let $c$ be a fixed element of $A$. Then for the binary operation $*$ defined by $x * y=f(x)+g(y)+c,(A, *)$ is a medial quasigroup.

Conversely, we have the Toyoda representation theorem [13].

Theorem 2.4. Let $(Q, *)$ be a medial quasigroup, then there exists a abelian group $(Q,+)$, two commuting automorphisms $f, g$ of $(Q,+)$ and a fixed element of $Q$ such that $x * y=f(x)+g(y)+c$.

Lemma 2.5. Let $A$ be an abelian group and $g$ be an automorphism of $A$. Then for an Alexander quandle $(A, *)$ defined by

$$
a * b=(I-g)(a)+g(b),(a, b \in A),
$$

where $I$ denotes the identity automorphism of $A$, the following statements are all equivalent.

(i) $(A, *)$ is a quasigroup.

(ii) $(A, *)$ is connected.

(iii) $f=I-g$ is an automorphism of $A$.

Proof. Implications $(i) \Rightarrow($ ii $)$ and $(i i i) \Rightarrow(i)$ are elementary.

$($ ii $) \Rightarrow($ iii $)$ : Let 0 be the neutral element of $(A,+)$. For an arbitrary element $b$ of $A$ consider an automorphism $\phi \in \operatorname{Inn}_{*}(A)$ such that $\phi(0)=b$. Then there exist $a_{1}, a_{2}, \cdots, a_{n} \in A$ such that $\phi=L_{a_{1}} L_{a_{2}} \cdots L_{a_{n}}$. Hence we have

$$
b=\phi(0)=a_{1} *\left(a_{2} * \cdots *\left(a_{n} * 0\right) \cdots\right) \in 0+f(A) .
$$

Thus $f$ is an epimorphism and hence an automorphism of $A$ since $A$ is finite.

Corollary 2.6. Any connected Alexander quandle is a medial idempotent quasigroup and vice versa.

Proof. It is easy to verify mediality and idempotency of an Alexander quandle. Hence one direction is followed from Lemma 2.5. From the Toyoda representation theorem and idempotency of $(Q, *)$ we have

$$
0=0 * 0=f(0)+g(0)+c ; c=0 .
$$

Then applying idempotency of $(Q, *)$ once more, we see that

$$
a=a * a=f(a)+g(a) \forall a \in Q ; f=I-g .
$$

Thus we have the other direction. 
The last part of this section is a brief introduction of Andruskiewitsch-Graña' extension theory of quandles by dynamical cocycles. For more details see [1].

For a quandle $(X, \cdot)$ and a non-empty set $S$, a function $\alpha: X \times X \rightarrow S^{S \times S}$ is said to be a dynamical cocycle if the following conditions hold for $\alpha_{x y}=\alpha(x, y)$ :

(i) $\alpha_{x x}(a, a)=a$ for all $x \in X$ and $a \in S$

(ii) $\alpha_{x y}(a,-): S \rightarrow S$ is bijection for all $x, y \in X$ and for all $a \in S$

(iii) $\alpha_{x(y \cdot z)}\left(a, \alpha_{y z}(b, c)\right)=\alpha_{(x \cdot y)(x \cdot z)}\left(\alpha_{x y}(a, b), \alpha_{x z}(a, c)\right)$ for all $x, y, z \in X$ and for all $a, b, c \in S$

Given a quandle $(X, \cdot)$ with a dynamical cocycle $\alpha$, by defining a binary operation $*$ on a set $X \times S$ so that

$$
(x, a) *(y, b)=\left(x \cdot y, \alpha_{x y}(a, b)\right) \forall(x, a),(y, b) \in X \times S
$$

we have a quandle which is called an extension of $X$ by a dynamical 2-cocycle $\alpha$ and denoted by $X \times{ }_{\alpha} S$.

For an extension $E=X \times{ }_{\alpha} S, X=(X, \cdot)$ is called the base (of $E$ ). And we have the natural projection $\pi: E \rightarrow X$ defined by $\pi(x, a)=x(x \in X, a \in S)$, which is a quandle homomorphism. Then for each $x \in X$ a subquandle $E_{x}=$ $\pi^{-1}(x)$ of $E$ is called a fiber (of $E$ ).

The following lemma may be utilized to see if a given quandle $(E, *)$ is extended from a quandle $(X, \cdot)$ by a dynamical cocycle $\alpha$.

Lemma 2.7. [1] Let $(E, *)$ be a quandle satisfying following conditions:

(i) $E$ is a disjoint union $E=\cup_{x \in X} E_{x}$ for a non-empty set $X$,

(ii) a binary operation $\cdot$ is defined on $X$ so that $E_{x} * E_{y}=E_{x \cdot y}$ for all $x, y \in X$,

(iii) $\left|\operatorname{card}\left(E_{x}\right)\right|=\left|\operatorname{card}\left(E_{y}\right)\right|$ for all $x, y \in X$.

Then $(X, \cdot)$ is a quandle. Furthermore, let $S$ be a set such that card $(S)=$ $\operatorname{card}\left(E_{x}\right)$ and take a bijection $g_{x}: E_{x} \rightarrow S$ for each $x \in X$. Then a function $\alpha: X \times X \rightarrow S^{S \times S}$ defined by

$$
\alpha_{x y}(a, b)=g_{x \cdot y}\left(g_{x}^{-1}(a) * g_{y}^{-1}(y)\right)
$$

is a dynamical cocycle and $E \cong X \times_{\alpha} S$

\section{Main results}

\subsection{A dynamical cocycle associated with the Galkin quandle $G_{15}$}

For examples of non-medial left-distributive quasigroups, in his survey article [5] Galkin considered a binary operation on a set $G_{3 p}=\mathbf{Z}_{p} \times \mathbf{Z}_{3}$ defined by

$$
(a, x) \circ(b, y)=(\mu(-x+y) a-b+\tau(-x+y),-x-y)
$$

where a function $\mu: \mathbf{Z}_{3} \rightarrow \mathbf{Z}_{5}$ is defined so that $\mu(x)=2$ for $x=0, \mu(x)=-1$ for $x \neq 0$, and a function $\tau: \mathbf{Z}_{3} \rightarrow \mathbf{Z}_{p}$ is defined so that $\tau(0)=0$. In particular he showed that for odd prime $p$, the above quandles are indeed non-medial left-distributive quasigroups, We call them Galkin quandles. One notices that the function $\tau$ is rather ambiguously defined. Unfortunately, we could not 
access to his unpublished Russian paper [6] possibly containing more accurate information regarding $\tau$. Indeed Clark and et al. [3] more rigorously denoted Galkin quandles by $G\left(Z_{p}, c_{1}, c_{2}\right)$ where $c_{i}=\tau(i)$ for $i=1,2$. Then they showed that $G\left(Z_{p}, c_{1}, c_{2}\right)$ is isomorphic to $G\left[Z_{p}, 0, c_{2}-c_{1}\right)$ which is denoted by $G\left(Z_{p}, c_{2}-c_{1}\right)$ for short. Furthermore they proved that up to isomorphism there are two Galkin quandles of order $15 ; G\left(Z_{5},[0]\right)$ and $G\left(Z_{5},[1]\right)$ where $[c]$ denotes the modulo class of $c$ with respect to $p$. In [2] we took $c_{1}=c_{2}=0$ for a Galkin quandle $G_{15}$ which corresponds to $G\left(Z_{5},[0]\right)$.

To see that $G_{15}$ is indeed an extension of the Tait quandle by a dynamical cocycle. We work with a multiplication table of $G_{15}$. Although our strategy for a proof of the following theorem is based on Lemma 2.7 we do not assume left-distributivity of $G_{15}$ for a self-contained proof. We derive a function $\alpha: X \times X \rightarrow S^{S \times S}$ from the multiplication table of $G_{15}$ and show that $\alpha$ is indeed a dynamical cocyle associated with an extension of the Tait quandle.

Theorem 3.1. Let $G_{15}=\left(\mathbf{Z}_{15}, *\right)$ be a quasigroup defined by a binary operation in TABLE 1. Then $G_{15}$ is an extension of the Tait quandle by a dynamical cocycle.

\begin{tabular}{|c|ccccc|ccccc|ccccc|}
\hline$*$ & 0 & 3 & 6 & 9 & 12 & 1 & 4 & 7 & 10 & 13 & 2 & 5 & 8 & 11 & 14 \\
\hline 0 & 0 & 12 & 9 & 6 & 3 & 2 & 14 & 11 & 8 & 5 & 1 & 13 & 10 & 7 & 4 \\
3 & 6 & 3 & 0 & 12 & 9 & 14 & 11 & 8 & 5 & 2 & 13 & 10 & 7 & 4 & 1 \\
6 & 12 & 9 & 6 & 3 & 0 & 11 & 8 & 5 & 2 & 14 & 10 & 7 & 4 & 1 & 13 \\
9 & 3 & 0 & 12 & 9 & 6 & 8 & 5 & 2 & 14 & 11 & 7 & 4 & 1 & 13 & 10 \\
12 & 9 & 6 & 3 & 0 & 12 & 5 & 2 & 14 & 11 & 8 & 4 & 1 & 13 & 10 & 7 \\
\hline 1 & 2 & 14 & 11 & 8 & 5 & 1 & 13 & 10 & 7 & 4 & 0 & 12 & 9 & 6 & 3 \\
4 & 14 & 11 & 8 & 5 & 2 & 7 & 4 & 1 & 13 & 10 & 12 & 9 & 6 & 3 & 0 \\
7 & 11 & 8 & 5 & 2 & 14 & 13 & 10 & 7 & 4 & 1 & 9 & 6 & 3 & 0 & 12 \\
10 & 8 & 5 & 2 & 14 & 11 & 4 & 1 & 13 & 10 & 7 & 6 & 3 & 0 & 12 & 9 \\
13 & 5 & 2 & 14 & 11 & 8 & 10 & 7 & 4 & 1 & 13 & 3 & 0 & 12 & 9 & 6 \\
\hline 2 & 1 & 13 & 10 & 7 & 4 & 0 & 12 & 9 & 6 & 3 & 2 & 14 & 11 & 8 & 5 \\
5 & 13 & 10 & 7 & 4 & 1 & 12 & 9 & 6 & 3 & 0 & 8 & 5 & 2 & 14 & 11 \\
8 & 10 & 7 & 4 & 1 & 13 & 9 & 6 & 3 & 0 & 12 & 14 & 11 & 8 & 5 & 2 \\
11 & 7 & 4 & 1 & 13 & 10 & 6 & 3 & 0 & 12 & 9 & 5 & 2 & 14 & 11 & 8 \\
14 & 4 & 1 & 13 & 10 & 7 & 3 & 0 & 12 & 9 & 6 & 11 & 8 & 5 & 2 & 14 \\
\hline
\end{tabular}

TABLE 1 : the Galkin quandle $G_{15}$

Proof. Let $E=G_{15}$ and $E_{x}=\{x, 3+x, 6+x, 9+x, 12+x\}$ for each $x \in \mathbf{Z}_{3}$. And take $X$ in Lemma 2.7 as the Tait quandle $R_{3}=\left(\mathbf{Z}_{3}, \cdot\right)$ which has a multiplication table: 


\begin{tabular}{l|lll} 
& 0 & 1 & 2 \\
\hline 0 & 0 & 2 & 1 \\
1 & 2 & 1 & 0 \\
2 & 1 & 0 & 2
\end{tabular}

TABLE 2: the Tait quandle $R_{3}$

Further let $S=\mathbf{Z}_{5}$ and take bijections $g_{x}: E_{x} \rightarrow S$ in Lemma 2.7 so that $g_{x}(x)=0, g_{x}(3+x)=1, g_{x}(6+x)=2, g_{x}(9+x)=3$ and $g_{x}(12+x)=4$. Then one can easily see that the condition (ii) of Lemma 2.7 holds for $G_{15}$. Moreover the function $\alpha: X \times X \rightarrow S^{S \times S}$ defined by $\alpha_{x y}(a, b)=g_{x \cdot y}\left(g_{x}^{-1}(a) * g_{y}^{-1}(y)\right)$ can be explicitly determined as shown in TABLE 3 .

\begin{tabular}{|c|c|c|c|}
\hline & $g_{0}^{-1}\left(E_{0}\right)$ & $g_{1}^{-1}\left(E_{1}\right)$ & $g_{2}^{-1}\left(E_{2}\right)$ \\
\hline$g_{0}^{-1}\left(E_{0}\right)$ & $\alpha_{00}(a, b)=2 a-b$ & $\alpha_{01}(a, b)=-a-b$ & $\alpha_{02}(a, b)=-a-b$ \\
\hline$g_{1}^{-1}\left(E_{1}\right)$ & $\alpha_{10}(a, b)=-a-b$ & $\alpha_{11}(a, b)=2 a-b$ & $\alpha_{12}(a, b)=-a-b$ \\
\hline$g_{2}^{-1}\left(E_{2}\right)$ & $\alpha_{20}(a, b)=-a-b$ & $\alpha_{21}(a, b)=-a-b$ & $\alpha_{22}(a, b)=2 a-b$ \\
\hline
\end{tabular}

TABLE 3

Note for instance that for each $x \in R_{3},\left(\mathbf{Z}_{5}, \alpha_{x x}\right)$ is $R_{5}$, the dihedral quandle of order 5 .

Now we show that $\alpha$ is indeed a dynamical cocycle. It is enough to check that the identity

$$
\alpha_{x(y \cdot z)}\left(a, \alpha_{y z}(b, c)\right)=\alpha_{(x \cdot y)(x \cdot z)}\left(\alpha_{x y}(a, b), \alpha_{x z}(a, c)\right)
$$

holds for every $x, y, z \in R_{3}$ and $a, b, c \in \mathbf{Z}_{5}$. Put the left and right hand side of above identity $(L H S)$ and $(R H S)$ respectively.

CASE 1: $x \neq y$ and $y \neq z$

$$
\begin{aligned}
(\text { LHS }) & =\alpha_{x x}(a,-b-c) \\
& =2 a+b+c \\
(R H S) & =\alpha_{x y}(-a-b,-a-c) \\
& =2 a+b+c
\end{aligned}
$$

CASE 2: $x \neq y$ and $y=z$

$$
\begin{aligned}
(\text { LHS }) & =\alpha_{x y}\left(a, \alpha_{y y}(b, c)\right) \\
& =\alpha_{x y}(a, 2 b-c) \\
& =-a-2 b+c \\
(R H S) & =\alpha_{(x \cdot y)(x \cdot y)}\left(\alpha_{x y}(a, b), \alpha_{x y}(a, c)\right) \\
& =\alpha_{(x \cdot y)(x \cdot y)}(-a-b,-a-c) \\
& =-a-2 b+c
\end{aligned}
$$


CASE 3: $x=y$ and $y \neq z$

$$
\begin{aligned}
(L H S) & =\alpha_{x(x \cdot z)}(a,-b-c) \\
& =-a+b+c \\
(R H S) & =\alpha_{x(x \cdot z)}\left(\alpha_{x x}(a, b), \alpha_{x z}(a, c)\right) \\
& =\alpha_{x(x \cdot z)}(2 a-b,-a-c) \\
& =-a+b+c
\end{aligned}
$$

CASE 4: $x=y=z$

$$
\begin{aligned}
(\text { LHS }) & =\alpha_{x x}\left(a, \alpha_{x x}(b, c)\right) \\
& =\alpha_{x x}(a, 2 b-c) \\
& =2 a-2 b+c \\
(R H S) & =\alpha_{x x}\left(\alpha_{x x}(a, b), \alpha_{x x}(a, c)\right) \\
& =\alpha_{x x}(2 a-b, 2 a-c) \\
& =2 a-2 b+c
\end{aligned}
$$

\subsection{A dynamical cocycle associated with the Stanovsky quandle $\boldsymbol{S}_{15}$}

We say that quasigroups $(Q, *)$ and $(R, \circ)$ are isotopic, if there are bijections $\alpha, \beta, \gamma: Q \rightarrow R$ such that $\alpha(x * y)=\beta(x) \circ \gamma(y)$ for all $x, y \in Q$. Then the Toyoda representation theorem says that a medial quasigroup $(Q, *)$ is isotopic to an abelian group $(Q,+)$.

Likewise, it is known that any distributive quasigroup $(Q, \cdot)$ is isotopic to a commutative Moufang loop $(Q, \circ)$ in such a way that

$$
a \circ b=L_{e}(a) \cdot R_{e}(b)
$$

where $e$ is a fixed element of $Q$, and $L_{e}, R_{e}$ are the left and right translation of $e$ respectively. As for a left-distributive quasigroup it is expected that it is isotopic to so called a Bol loop. But Stanovsky [11] came up with a counterexample to this idea by using a computer as shown in TABLE 4.

His example attracts our attentions because of its non-mediality. Through quandle isomorphisms, we can transform TABLE 4 into TABLE 5 which may be thought of as a kind of a normalized multiplication table for an extension of the Tait quandle with fiber isomorphic to $R_{5}$ as in the case of the Galkin quandle $G_{15}$. 


\begin{tabular}{c|ccccccccccccccc}
$*$ & 0 & 1 & 2 & 3 & 4 & 5 & 6 & 7 & 8 & 9 & 10 & 11 & 12 & 13 & 14 \\
\hline 0 & 0 & 2 & 4 & 1 & 6 & 3 & 8 & 5 & 10 & 7 & 9 & 12 & 11 & 14 & 13 \\
1 & 3 & 1 & 0 & 11 & 5 & 4 & 12 & 14 & 9 & 8 & 13 & 7 & 2 & 6 & 10 \\
2 & 1 & 12 & 2 & 6 & 0 & 13 & 3 & 10 & 11 & 14 & 7 & 4 & 5 & 9 & 8 \\
3 & 5 & 0 & 7 & 3 & 11 & 12 & 10 & 2 & 14 & 13 & 6 & 1 & 9 & 8 & 4 \\
4 & 2 & 8 & 11 & 14 & 4 & 9 & 0 & 13 & 1 & 5 & 12 & 3 & 6 & 10 & 7 \\
5 & 7 & 9 & 12 & 0 & 8 & 5 & 13 & 11 & 4 & 1 & 14 & 10 & 3 & 2 & 6 \\
6 & 4 & 13 & 10 & 7 & 12 & 14 & 6 & 3 & 0 & 11 & 2 & 8 & 1 & 5 & 9 \\
7 & 9 & 11 & 6 & 10 & 14 & 0 & 2 & 7 & 13 & 12 & 3 & 5 & 8 & 4 & 1 \\
8 & 6 & 5 & 14 & 13 & 9 & 1 & 11 & 12 & 8 & 4 & 0 & 2 & 10 & 7 & 3 \\
9 & 10 & 4 & 13 & 12 & 1 & 8 & 14 & 0 & 5 & 9 & 11 & 6 & 7 & 3 & 2 \\
10 & 8 & 14 & 3 & 2 & 13 & 11 & 7 & 6 & 12 & 0 & 10 & 9 & 4 & 1 & 5 \\
11 & 14 & 3 & 8 & 4 & 2 & 7 & 9 & 1 & 6 & 10 & 5 & 11 & 13 & 12 & 0 \\
12 & 13 & 6 & 1 & 5 & 10 & 2 & 4 & 9 & 7 & 3 & 8 & 14 & 12 & 0 & 11 \\
13 & 11 & 10 & 5 & 9 & 7 & 6 & 1 & 8 & 3 & 2 & 4 & 0 & 14 & 13 & 12 \\
14 & 12 & 7 & 9 & 8 & 3 & 10 & 5 & 4 & 2 & 6 & 1 & 13 & 0 & 11 & 14
\end{tabular}

TABLE 4

Theorem 3.2. Let $S_{15}=\left(\mathbf{Z}_{15}, *\right)$ be a quasigroup defined by a binary operation in TABLE 5. Then $S_{15}$ is an extension of the Tait quandle by a dynamical cocycle.

\begin{tabular}{|c|ccccc|ccccc|ccccc|}
\hline$*$ & 0 & 3 & 6 & 9 & 12 & 1 & 4 & 7 & 10 & 13 & 2 & 5 & 8 & 11 & 14 \\
\hline 0 & 0 & 12 & 9 & 6 & 3 & 2 & 11 & 5 & 14 & 8 & 10 & 1 & 7 & 13 & 4 \\
3 & 6 & 3 & 0 & 12 & 9 & 5 & 14 & 8 & 2 & 11 & 4 & 10 & 1 & 7 & 13 \\
6 & 12 & 9 & 6 & 3 & 0 & 8 & 2 & 11 & 5 & 4 & 13 & 4 & 10 & 1 & 7 \\
9 & 3 & 0 & 12 & 9 & 6 & 11 & 5 & 4 & 8 & 2 & 7 & 13 & 4 & 10 & 1 \\
12 & 9 & 6 & 3 & 0 & 12 & 14 & 8 & 2 & 11 & 5 & 1 & 7 & 13 & 4 & 10 \\
\hline 1 & 5 & 8 & 11 & 14 & 2 & 1 & 13 & 10 & 7 & 4 & 0 & 3 & 6 & 9 & 12 \\
4 & 14 & 2 & 5 & 8 & 11 & 7 & 4 & 1 & 13 & 10 & 6 & 9 & 12 & 0 & 3 \\
7 & 8 & 11 & 14 & 2 & 5 & 13 & 10 & 7 & 4 & 1 & 12 & 0 & 3 & 6 & 9 \\
10 & 2 & 5 & 8 & 11 & 14 & 4 & 1 & 13 & 10 & 7 & 3 & 6 & 9 & 12 & 0 \\
13 & 11 & 14 & 2 & 5 & 8 & 10 & 7 & 4 & 1 & 13 & 9 & 12 & 0 & 3 & 6 \\
\hline 2 & 1 & 10 & 4 & 13 & 7 & 12 & 3 & 9 & 0 & 6 & 2 & 14 & 11 & 8 & 5 \\
5 & 7 & 1 & 10 & 4 & 13 & 0 & 6 & 12 & 3 & 9 & 8 & 5 & 2 & 14 & 11 \\
8 & 13 & 7 & 1 & 10 & 4 & 3 & 9 & 0 & 6 & 12 & 14 & 11 & 8 & 5 & 2 \\
11 & 4 & 13 & 7 & 1 & 10 & 6 & 12 & 3 & 9 & 0 & 5 & 2 & 14 & 11 & 8 \\
14 & 10 & 4 & 13 & 7 & 1 & 9 & 0 & 6 & 12 & 3 & 11 & 8 & 5 & 2 & 14 \\
\hline
\end{tabular}

TABLE 5: the Stanovsky quandle $S_{15}$

Proof. With the notations in the proof of Theorem 3.1, from TABLE 5 we can come up with a function $\alpha: X \times X \rightarrow S^{S \times S}$ as shown in TABLE 6 . 


\begin{tabular}{|c|c|c|c|}
\hline & $g_{0}^{-1}\left(E_{0}\right)$ & $g_{1}^{-1}\left(E_{1}\right)$ & $g_{2}^{-1}\left(E_{2}\right)$ \\
\hline$g_{0}^{-1}\left(E_{0}\right)$ & $\alpha_{00}(a, b)=2 a-b$ & $\alpha_{01}(a, b)=a+3 b$ & $\alpha_{02}(a, b)=3 a+2 b+3$ \\
\hline$g_{1}^{-1}\left(E_{1}\right)$ & $\alpha_{10}(a, b)=3 a+b+1$ & $\alpha_{11}(a, b)=2 a-b$ & $\alpha_{12}(a, b)=2 a+b$ \\
\hline$g_{2}^{-1}\left(E_{2}\right)$ & $\alpha_{20}(a, b)=2 a+3 b$ & $\alpha_{21}(a, b)=a+2 b+4$ & $\alpha_{22}(a, b)=2 a-b$ \\
\hline
\end{tabular}

TABLE 6

The following rutin calculations show that $\alpha$ is indeed a dynamical cocycle.

CASE I) $x \neq y, y \neq z$

(1) $x=0, y=1, z=2: \alpha_{0(1 \cdot 2)}\left(a, \alpha_{12}(b, c)\right)=\alpha_{(0 \cdot 1)(0 \cdot 2)}\left(\alpha_{01}(a, b), \alpha_{02}(a, c)\right)$

$$
\begin{aligned}
(L H S) & =\alpha_{00}(a, 2 b+c) \\
& =2 a+3 b+4 c\left(\text { in } \mathbf{Z}_{5}\right) \\
(R H S) & =\alpha_{21}(a+3 b, 3 a+2 c+3) \\
& =2 a+3 b+4 c\left(\text { in } \mathbf{Z}_{5}\right)
\end{aligned}
$$

(2) $x=0, y=2, z=1: \alpha_{0(2 \cdot 1)}\left(a, \alpha_{21}(b, c)\right)=\alpha_{(0 \cdot 2)(0 \cdot 1)}\left(\alpha_{02}(a, b), \alpha_{01}(a, c)\right)$

$$
\begin{aligned}
(\text { LHS }) & =\alpha_{00}(a, b+2 c+4) \\
& =2 a+4 b+3 c+1\left(\text { in } \mathbf{Z}_{5}\right) \\
(R H S) & =\alpha_{12}(3 a+2 b+3, a+3 c) \\
& =2 a+4 b+3 c+1\left(\text { in } \mathbf{Z}_{5}\right)
\end{aligned}
$$

(3) $x=1, y=0, z=2: \alpha_{1(0 \cdot 2)}\left(a, \alpha_{02}(b, c)\right)=\alpha_{(1 \cdot 0)(1 \cdot 2)}\left(\alpha_{10}(a, b), \alpha_{12}(a, c)\right)$

$$
\begin{aligned}
(\text { LHS }) & =\alpha_{11}(a, 3 b+2 c+3) \\
& =2 a+2 b+3 c+2\left(\text { in } \mathbf{Z}_{5}\right) \\
(R H S) & =\alpha_{20}(3 a+b+1,2 a+c) \\
& =2 a+2 b+3 c+2\left(\text { in } \mathbf{Z}_{5}\right)
\end{aligned}
$$

(4) $x=1, y=2, z=0: \alpha_{1(2 \cdot 0)}\left(a, \alpha_{20}(b, c)\right)=\alpha_{(1 \cdot 2)(1 \cdot 0)}\left(\alpha_{12}(a, b), \alpha_{10}(a, c)\right)$

$$
\begin{aligned}
(L H S) & =\alpha_{11}(a, 2 b+3 c) \\
& =2 a+3 b+2 c\left(\text { in } \mathbf{Z}_{5}\right) \\
(R H S) & =\alpha_{02}(2 a+b, 3 a+c+1) \\
& =2 a+3 b+2 c\left(\text { in } \mathbf{Z}_{5}\right)
\end{aligned}
$$

(5) $x=2, y=0, z=1: \alpha_{2(0 \cdot 1)}\left(a, \alpha_{01}(b, c)\right)=\alpha_{(2 \cdot 0)(2 \cdot 1)}\left(\alpha_{20}(a, b), \alpha_{21}(a, c)\right)$

$$
\begin{aligned}
(L H S) & =\alpha_{22}(a, b-2 c) \\
& =2 a+4 b+2 c\left(\text { in } \mathbf{Z}_{5}\right) \\
(R H S) & =\alpha_{10}(2 a+3 b, a+2 c+4) \\
& =2 a+4 b+2 c\left(\text { in } \mathbf{Z}_{5}\right)
\end{aligned}
$$


(6) $x=2, y=1, z=0: \alpha_{2(1 \cdot 0)}\left(a, \alpha_{10}(b, c)\right)=\alpha_{(2 \cdot 1)(2 \cdot 0)}\left(\alpha_{21}(a, b), \alpha_{20}(a, c)\right)$

$$
\begin{aligned}
(\text { LHS }) & =\alpha_{22}(a, 3 b+c+1) \\
& =2 a+2 b+4 c+4\left(\text { in } \mathbf{Z}_{5}\right) \\
(\text { RHS }) & =\alpha_{01}(a+2 b+4,2 a+3 c) \\
& =2 a+2 b+4 c+4\left(\text { in } \mathbf{Z}_{5}\right)
\end{aligned}
$$

CASE II) $x=y, y \neq z$

(1) $x=y=0, z=1: \alpha_{0(0 \cdot 1)}\left(a, \alpha_{01}(b, c)\right)=\alpha_{(0 \cdot 0)(0 \cdot 1)}\left(\alpha_{00}(a, b), \alpha_{01}(a, c)\right)$

$$
\begin{aligned}
(\text { LHS }) & =\alpha_{02}(a, b+3 c) \\
& =3 a+2 b+c+3\left(\text { in } \mathbf{Z}_{5}\right) \\
(\text { RHS }) & =\alpha_{02}(2 a-b, a+3 c) \\
& =3 a+2 b+c+3\left(\text { in } \mathbf{Z}_{5}\right)
\end{aligned}
$$

(2) $x=y=0, z=2: \alpha_{0(0 \cdot 2)}\left(a, \alpha_{02}(b, c)\right)=\alpha_{(0 \cdot 0)(0 \cdot 2)}\left(\alpha_{00}(a, b), \alpha_{02}(a, c)\right)$

$$
\begin{aligned}
(L H S) & =\alpha_{01}(a, 3 b+2 c+3) \\
& =a+4 b+c+4\left(\text { in } \mathbf{Z}_{5}\right) \\
(R H S) & =\alpha_{01}(2 a-b, 3 a+2 c+3) \\
& =a+4 b+c+4\left(\text { in } \mathbf{Z}_{5}\right)
\end{aligned}
$$

(3) $x=y=1, z=0: \alpha_{1(1 \cdot 0)}\left(a, \alpha_{10}(b, c)\right)=\alpha_{(1 \cdot 1)(1 \cdot 0)}\left(\alpha_{11}(a, b), \alpha_{10}(a, c)\right)$

$$
\begin{aligned}
(\text { LHS }) & =\alpha_{12}(a, 3 b+c+1) \\
& =2 a+3 b+c+1\left(\text { in } \mathbf{Z}_{5}\right) \\
(R H S) & =\alpha_{12}(2 a-b, 3 a+c+1) \\
& =2 a+3 b+c+1\left(\text { in } \mathbf{Z}_{5}\right)
\end{aligned}
$$

(4) $x=y=1, z=2: \alpha_{1(1 \cdot 2)}\left(a, \alpha_{12}(b, c)\right)=\alpha_{(1 \cdot 1)(1 \cdot 2)}\left(\alpha_{11}(a, b), \alpha_{12}(a, c)\right)$

$$
\begin{aligned}
(L H S) & =\alpha_{10}(a, 2 b+c) \\
& =3 a+2 b+c+1\left(\text { in } \mathbf{Z}_{5}\right) \\
(R H S) & =\alpha_{10}(2 a-b, 2 a+c) \\
& =3 a+2 b+c+1\left(\text { in } \mathbf{Z}_{5}\right)
\end{aligned}
$$

(5) $x=y=2, z=0: \alpha_{2(2 \cdot 0)}\left(a, \alpha_{20}(b, c)\right)=\alpha_{(2 \cdot 2)(2 \cdot 0)}\left(\alpha_{22}(a, b), \alpha_{20}(a, c)\right)$

$$
\begin{aligned}
(L H S) & =\alpha_{21}(a, 2 b+3 c) \\
& =a+4 b+c+4\left(\text { in } \mathbf{Z}_{5}\right) \\
(R H S) & =\alpha_{21}(2 a-b, 2 a+3 c) \\
& =a+4 b+c+4\left(\text { in } \mathbf{Z}_{5}\right)
\end{aligned}
$$


(6) $x=y=2, z=1: \alpha_{2(2 \cdot 1)}\left(a, \alpha_{21}(b, c)\right)=\alpha_{(2 \cdot 2)(2 \cdot 1)}\left(\alpha_{22}(a, b), \alpha_{21}(a, c)\right)$

$$
\begin{aligned}
(\text { LHS }) & =\alpha_{20}(a, b+2 c+4) \\
& =2 a+3 b+c+2\left(\text { in } \mathbf{Z}_{5}\right) \\
(\text { RHS }) & =\alpha_{20}(2 a-b, a+2 c+4) \\
& =2 a+3 b+c+2\left(\text { in } \mathbf{Z}_{5}\right)
\end{aligned}
$$

CASE III $) \quad x=y=z: \alpha_{x(x \cdot x)}\left(a, \alpha_{x x}(b, c)\right)=\alpha_{(x \cdot x)(x \cdot x)}\left(\alpha_{x x}(a, b), \alpha_{x x}(a, c)\right)$

$$
\begin{aligned}
(L H S) & =\alpha_{x x}(a, 2 b-c) \\
& =2 a+3 b+c\left(\text { in } \mathbf{Z}_{5}\right) \\
(R H S) & =\alpha_{x x}(2 a-b, 2 a-c) \\
& =2 a+3 b+c\left(\text { in } \mathbf{Z}_{5}\right)
\end{aligned}
$$

Using a computer programming GAP, Vendramin [14] classified connected quandles of orders $\leq 32$, The $j$-th quandle of order $i$ is denoted by $C[i, j]$.

Theorem 3.3. The quandle $G_{15}$ is isomorphic to $C[15,6]=G\left(Z_{5},[0]\right)$, and the quandle $S_{15}$ is isomorphic to $C[15,5]=G\left(Z_{5},[1]\right)$.

Proof. There are 7 connected quandles of order 15 consisting of 3 Alexander quandles: $C[15,1]=Z_{15}[t] /(t+1), C[15,3]=Z_{15}[t] /(t+7), C[15,4]=$ $Z_{15}[t] /(t+13)$, two non-medial quasigroups $C[15,6]=G\left(Z_{5},[0]\right)$ and $C[15,5]=$ $G\left(Z_{5},[1]\right)$ and two non-medial, non-quasigroups $C[15,2], C[15,7]$. Furthermore $C[15,6]=G\left(Z_{5},[0]\right)$ is involutive but $C[15,5]=G\left(Z_{5},[1]\right)$ is not. Thus the claims are followed from the following observations (a) and (b):

(a) Non-medialty of $G_{15}$ and $S_{15}$ : Take $x=0, y=3, z=1$ and $w=2$. Then from TABLE 1 of $G_{15}$, we have

$(x * y) *(z * w)=(0 * 3) *(1 * 2)=9 \neq 3=(0 * 1) *(3 * 2)=(x * z) *(y * w)$

Likewise from TABLE 5 of $S_{15}$, we have

$(x * y) *(z * w)=(0 * 3) *(1 * 2)=9 \neq 3=(0 * 1) *(3 * 2)=(x * z) *(y * w)$.

(b) Isomorphic invariance of types of cycles in the disjoint cyclic decomposition of a left-translation [9]: Indeed each left-translation of $G_{15}$ is an involution consisting of 7-disjoint transpositions whereas that of $S_{15}$ consists of a single 10-cycle and two transpositions which are mutually disjoint.

\section{References}

[1] N. Andruskiewitsch and M. Graña, From racks to pointed Hopf algebras, Adv. Math. 178(2) (2003), 177-243. Also in math.QA/0202084.

[2] Y.-G. Baik, H.-S. Sim and H.-J. Song, Connected quandles of small orders, unpublished manuscript 2008.

[3] W. Clark, M. Elhamdadi, X. Hou, M. Saito and T. Yeyman, Connected quandles Associated with Pointed Abelian Groups, arXiv:11107.5777.

[4] M. Elhamdadi, Distributivity in Quandles and Quasigroups, arXiv:1209.6518. 
[5] V.M. Galkin, Quasigroups, J. Soviet Math. 49 (1990), no. 3, 941-967.

[6] V.M. Galkin, Left-distributive finite order quasigroups, (Russian) Quasigroups and loops. Mat. Issled. (1979), no. 51, 43-54, 163.

[7] D. Joice, A classifying invariants of knots, the knot quandles, J. Pure Appl. Alg. 23 (1982) 37-65.

[8] T. Kepka and P. Nemec, Commutative Moufang loops and distributive groupoids of small orders, Czechoslovak Math. J. 31(106) (1981), no. 4, 633-669.

[9] P. Lopes and D. Roseman, On Finite Racks and Quandles, arXiv:math/0412487v1 [math.GT] 24 Dec 24.

[10] J.-P. Soublin, Etude algebique de la notion de moyenne(suite et fin), J. Math. Pures Appl.(9) 50 (1971), 193-264.

[11] D. Stanovsky, Left distributive left quasigroups, $\mathrm{PhD}$ thesis, Charles Univ. Prague, 2004.

[12] D.S. Siver and S.G. Williams, Generalized n-colorings of links, Banach Center Publications 42 (1998) Knot Theory, 381-394.

[13] K. Toyoda, On axioms of linear functions, Proc. Imp. Acad. Tokyo 17 (1941), 221-227.

[14] L. Vendramin, On the classification of quandles of low order, J. Knot Theory ramifications 21 (2012), no. 9, 1250088, 10 p.

[15] J. Vlach'y, Small left distributive quasigroups, Thesis, 2010.

HYUN-JONG SONG

Department of Applied Mathematics, Pukyong National University, Pusan 608737, KOREA

E-mail address: hjsong@pknu.ac.kr

Нyо-Sеов Sim

Department of Applied Mathematics, Pukyong National University, Pusan 608737, KOREA

E-mail address: hsim@pknu.ac.kr 